\title{
Quelle belle aventure ! Merci.
}

\section{Nicole Rege Colet}

\section{(2) OpenEdition}

\section{Journals}

Édition électronique

URL : http://journals.openedition.org/ripes/1109

DOI : 10.4000/ripes.1109

ISSN : 2076-8427

\section{Éditeur}

Association internationale de pédagogie universitaire

Référence électronique

Nicole Rege Colet, «Quelle belle aventure ! Merci. », Revue internationale de pédagogie de l'enseignement supérieur [En ligne], 32(2) | 2016, mis en ligne le 04 juillet 2016, consulté le 25 septembre 2020. URL : http://journals.openedition.org/ripes/1109; DOI : https://doi.org/10.4000/ripes.1109

Ce document a été généré automatiquement le 25 septembre 2020.

Licence CC BY-NC-SA 


\title{
Quelle belle aventure! Merci.
}

\author{
Nicole Rege Colet
}

1 En 2008, l'assemblée générale de l'Association internationale de pédagogie universitaire me confiait la rédaction de la revue officielle de l'AIPU qui s'appelait, à cette époque, Res Academica. Il s'agissait non seulement de reprendre le flambeau de l'équipe éditoriale de l'Université de Sherbrooke, sous la direction de Jean-François Desbiens, mais aussi d'effectuer la transition - innovation oblige - vers une version en ligne. En effet, à Montpellier, nous avons décidé de faciliter la diffusion des recherches et des retours d'expériences de la communauté des protagonistes de la pédagogie de l'enseignement supérieur et de favoriser l'accès aux productions scientifiques de qualité publiées en français.

2 Les premiers mois de mon mandat ont été occupés à préparer le dossier pour être hébergé sur une plateforme de qualité et à trouver un nouveau nom pour la revue. Res Academica est ainsi devenue la Revue internationale de pédagogie de l'enseignement supérieur, marquant une évolution terminologique importante, celle de la pédagogie universitaire à la pédagogie de l'enseignement supérieur, un nouveau nom qui souligne l'approche inclusive voulue par la communauté de l'AIPU. Notre dossier a été retenu par le comité de sélection de revues.org et le premier numéro de RIPES a été publié en avril 2009. Il porte le numéro 25(1) pour marquer la continuité avec Res Academica qui s'est arrêtée à 24(2).

3 Depuis, ce sont 18 numéros qui ont été publiés. Au départ, nous visions deux numéros par an. Peu à peu, RIPES s'est fait une place dans le monde de la publication scientifique francophone : elle est reconnue pour sa qualité scientifique et a fait l'objet d'un audit. La collection compte plusieurs numéros thématiques qui font suite à des symposiums ou des congrès. Ainsi, depuis deux ans, ce sont trois numéros par an qui sont publiés, preuve en est de la vitalité grandissante de la revue. La transition vers le numérique a apporté de la visibilité et rehaussé la qualité des productions : le défi lancé en mai 2008 a été pleinement rempli et l'AIPU peut se féliciter de ce travail collectif.

4 Il y a deux ans, lors de l'assemblée générale de l'AIPU à Mons, j'ai fait part de mon désir de me retirer progressivement de l'édition de RIPES. Je menais le navire depuis six ans et j'estimais qu'il était temps de passer le témoin à d'autres. Il en a toujours été ainsi au 
sein de l'AIPU afin que la revue ne soit pas trop fortement identifiée à une personne et qu'elle appartienne bien à la communauté, en incarnant le leadership distribué qui nous caractérise. A cet effet, j'avais déjà contacté Christelle Lison de l'Université de Sherbrooke qui s'est empressée de relever le défi quand elle a su que je lui proposais une période de co-direction pendant deux années pour assurer une transition en douceur.

La transmission des responsabilités éditoriales à Christelle Lison a été confirmée lors de l'assemblée générale de l'AIPU de juin 2016 à Lausanne. Le cérémonial m'a valu de belles émotions alors qu'Amaury Daele se répandait en éloges par rapport à mes talents d'amie critique. Je sais que certaines personnes se sont surtout frottées à la " critique "; j'espère qu'elles ont aussi pu y voir l'autre part, l'" amie ». Je suis repartie avec un magnifique crayon Caran d'Ache qui m'accompagnera dans mes prochains voyages dans l'univers fascinant de la facilitation graphique et une promesse faite à Christelle, celle de rédiger un dernier éditorial pour ce numéro 32(2).

6 Me voici, toujours fidèle, au rendez-vous ; je suis très fière de présenter les sept articles qui composent ce numéro. En les parcourant, j'ai constaté à quel point ils reflètent les thèmes qui animent la communauté de l'AIPU depuis ses débuts: l'évaluation, les pratiques d'enseignement innovantes et les dimensions psychologiques à travailler pour soutenir les apprentissages des étudiants comme la motivation et le sentiment d'auto-efficacité. Quatre équipes d'auteurs nous viennent du Québec - un effet du retour de la revue au Québec ? -, deux de la France et une de la Belgique. Mais où sont donc les auteurs suisses? Sans doute pris par l'organisation du $29^{\mathrm{e}}$ congrès de l'AIPU à Lausanne, ils ont délaissé leurs claviers pour nous organiser un congrès exceptionnel où nous avons examiné et discuté des valeurs dans l'enseignement supérieur. Manquent aussi les auteurs du continent africain. Ils ne sont pas loin et il est à souhaiter que le prochain congrès de l'AIPU, qui se tiendra au Bénin, inspirera de nouvelles contributions.

7 Valérie Wathelet de l'Université catholique de Louvain en Belgique, entourée par une équipe de pointe associant des collègues de l'Université de Namur et de l'Université catholique de Louvain, étudie l'auto-évaluation chez les étudiants de première année. Elle examine les estimations des étudiants quant à leur degré de maîtrise des prérequis à l'entrée afin d'en savoir plus sur leurs stratégies et leurs capacités à s'auto-évaluer avec pertinence et justesse.

Gilles Pinte de l'Université de Bretagne Sud s'intéresse au dispositif français de validation des acquis de l'expérience (VAE) et à ses effets pour le développement professionnel des candidats. Il présente les résultats d'une enquête conduite auprès de 223 étudiants ayant bénéficié d'une VAE pour savoir ce qu'ils sont devenus: les résultats sont pour le moins contrastés.

9 Louise Ménard et Diane Leduc, toutes les deux de l'Université de Québec à Montréal, sont connues pour leurs travaux sur la motivation des étudiants. Dans leur article, elles s'intéressent à celle relative aux enseignements de français et de littérature, des cours connaissant des forts taux d'abandon. Elles observent chez 1105 étudiants un décroissement de la motivation en cours d'année et identifient les pratiques d'enseignement susceptibles de soutenir cette motivation.

10 Olivia Monfette, de l'Université du Québec à Trois-Rivières, et Johanne Grenier, de l'Université du Québec à Montréal, abordent le sentiment d'auto-efficacité. Combinant enquête et entretiens, elles examinent l'évolution du sentiment d'auto-efficacité chez 
des étudiants en éducation psychique et à la santé. Elles confirment que les situations d'apprentissage authentique, telles que proposées pendant les stages, contribuent au développement du sentiment d'auto-efficacité.

11 Caroline Ladage de l'Université Aix-Marseille étudie les enseignements hybrides qui combinent cours en présence et cours à distance. Avec une méthode plurielle, elle évalue les effets d'un dispositif innovant tant sur la relation pédagogique et l'autonomisation des étudiants que sur leurs apprentissages et leur engagement dans les activités proposées.

Evaluer les apprentissages à distance reste un défi majeur. Isabelle Nizet, Julie Lyne Leroux, Colette Deaudelin et Jean Goulet de l'Université de Sherbrooke auquel se joint Sébastien Béland de l'Université de Montréal proposent un bilan des pratiques évaluatives dans des contextes d'enseignement à distance. Leurs travaux passent en revue les pratiques évaluatives mises en place à l'Université de Sherbrooke: ils en dégagent la diversité et formulent quelques recommandations pour un encadrement institutionnel de l'évaluation des apprentissages à distance.

13 Finalement, Elisabeth Martin, Catherine Lefrançois, Anne Guichard, Diane Tapp et Louise Arsenault, toutes de l'Université de Laval, présentent une grille d'évaluation critériée développée pour l'évaluation de productions écrites complexes au deuxième et troisième cycle. Elles relatent leur collaboration entre enseignantes, étudiante et conseillères pédagogiques pour élaborer et tester la grille de même que la démarche réflexive qu'a suscité cette collaboration inédite.

Il me reste quelques lignes pour formuler mes remerciements, tout d'abord, à tous les auteurs qui m'ont fait confiance et qui ont accepté de me croiser dans mon rôle d'amie critique. Merci aussi aux nombreux évaluateurs qui ont accepté de lire et de commenter les textes soumis, parfois dans des délais très courts. Leurs lectures attentives et leurs suggestions éclairées ont largement contribué au succès de RIPES. Et non des moindres, un tout grand merci à Brigitte Gourdange de l'Université de Liège qui s'occupe de la publication des textes. Brigitte est la personne sans qui RIPES n'existerait pas et nous lui devons beaucoup. Sans jamais perdre son calme, elle réceptionne les versions éditées et les met en ligne. Chaque fois qu'elle nous envoie pour validation la version finale, nous savons que le résultat sera parfait et que nous allons pouvoir donner aussitôt le feu vert pour publication.

Bons vents à toi Christelle ! Je te souhaite autant de plaisir que moi à naviguer le bateau de RIPES et à l'emmener vers de belles découvertes en matière de pédagogie de l'enseignement supérieur.

16 Strasbourg, le 4 juillet 2016

Nicole Rege Colet 


\section{AUTEUR}

NICOLE REGE COLET

Strasbourg 